\author{
${ }^{1 *}$ Kunakov S.K., ${ }^{2}$ Son E.E., ${ }^{1}$ Bolatov Zh., ${ }^{1}$ Kaster M. \\ ${ }^{1}$ International IT University, Almaty, Kazakhstan \\ ${ }^{2}$ Joint Institute of High Temperature, Moscow, Russian Federation \\ "e-mail: sandybeck.kunakov@gmail.com
}

\title{
Optical spectra in helium plasma generated by nuclear fission fragments
}

\begin{abstract}
Spectral study of optical radiation plasma formed by the products of nuclear reactions has some definite feature (unlike many other works on the irradiation of materials in the reactor) that experimental measurements should be carried out in a reactor at power. Problems associated with such types of experimental works are to provide biological protection with minimal loss of light emission and the difficulties of registration of the spectrum in the active zone of nuclear reactor. The first problem is solvable by choosing a specific design of the experimental setup; the second allows us to solve the photoelectric method of recording optical radiation, particularly using a photon counting technique.
\end{abstract}

Key words: helium plasma, optical characteristics, optical radiation, electron concentration, nuclearinduced plasma.

\section{Introduction}

In the reactor radiation, many optical materials reduce their capacity, and reflection ability due to the increase absorbency. It is therefore very important to ensure consistency in the process of installation of the optical properties, as well as to save needed transparency of the output window of the cartridge. During the measurement of the optical output radiation the biological protection can be achieved in different ways. Its implementation is based on the difference in interaction with matter of the short wave radiation of reactor and "soft" (hv 0.3 $-3 \mathrm{ev}$ ) optical radiation. To reflect the optical radiation from specific materials (mirror surfaces) some property of optical materials can be used, while a rigid reactor radiation is absorbed in this material, or passes there through. Usually in spectral diagnostics ampoule of cerium glass window are taken as a working material. The importance of such work (measurements during irradiation) has been repeatedly emphasized [1, 2]. If you do not perform spectral processing, the registered (recorded) spectrum will be distorted with respect to the emission spectrum. The spectral function of the experimental produce the corrected spectra (absolute spectra). At first step the information about the plasma can be ob- tained from the uncorrected spectra. In, particular, from the recorded spectra we can determine which atoms and molecules are displayed. Furthermore, by examining the intensity of radiation from the level of power deposited in the gas it can be traced in the changes in the spectrum, which depend on the chemical composition of the plasma. But it should be noted that the analysis of the recorded spectra brings for us only chance to compare the results in one optical line, and it is impossible to compare the intensity of different lines. The transition to the relative spectrum (taking into account the spectral function setting) allows comparing of the intensities of different lines and thereby obtaining much more information in the measurements of the spectral intensity distributions and compared with theoretical calculations. Also major confounding factors are associated with optical radiation from the reactor (output window ampoule fiber with a mirror), its analysis (monochromator), registration (PMT) there are a number of additional. Additional confounders include: a) irreversible changes in chemical composition ampoule b) emission of air in the diagnostic channel. The ratio of useful / spurious signals corresponds approximately to the ratio of energy input, the radiation from the channel is a set of molecular bands (most of nitrogen) emissions to air pretty 
strong continuum. Although this radiation is small it should be taken into account when measuring weak continuum from the plasma of the mixtures, c) glass ampoules luminescence (luminescence glass doped with cerium depends on the oxidation state; luminesce a glass containing in its composition trivalent cerium ions). Absorption bands located in trivalent cerium are active in the band of 310-320 nm. Most opportunities are opening absolute spectra. It is the absolute spectra which make it possible to study the conversion of nuclear energy into light and find the conversion factor of the level population, settlement and streams emptying different states (including ionization and recombination currents. In nuclear induced plasmas the formation of excited states of neutrals depends on the following two fluxes. The first one is the recombination processes and the second is the direct excitation by primary electrons and fission fragments. The electrons energy distribution evolves to its stationary state by its strong dependence from by the function of energy distribution of primary electrons, creates by collisions with neutrals and fission fragments excited helium states and its further formation directed by the structure of medium. The helium spectra measured in nuclear experiments on the test stationary reactor WWR-K by one of the authors (Kunakov Sandybeck) of the paper is compared with theoretical intensities obtained from the helium kinetics, where primary electrons as well as fission fragments are forming the optical spectra of the helium plasma.

\section{Optical spectra of helium plasma created by the fission fragments}

In order to ensure uniform volume ionization is necessary to choose in the reactor core area with a uniform distribution of the neutron flux. The main requirements for the nuclear reactions taking place under the action of thermal neutrons to form nuclear induced plasma, are sufficiently large cross sections of these reactions, and most released in the reaction energy. Among these the following reaction was applied to generate the helium-3 plasma as a volume source of ionization:

$$
{ }^{3} \mathrm{He}+n \rightarrow{ }^{3} \mathrm{H}+p+0.76 \mathrm{Mev},
$$

where $H e$ - helium, $n$ - neutron, $p$ - proton, $H-$ hydrogen.

The reaction cross section (0.1) for thermal neutrons is 5400 barns, and with increasing energy va- ries as $E^{1 / 2}$. The energy released is as follows: the nucleus of tritium - $578 \mathrm{keV}$ and $192 \mathrm{keV}$ - the energy of the proton.

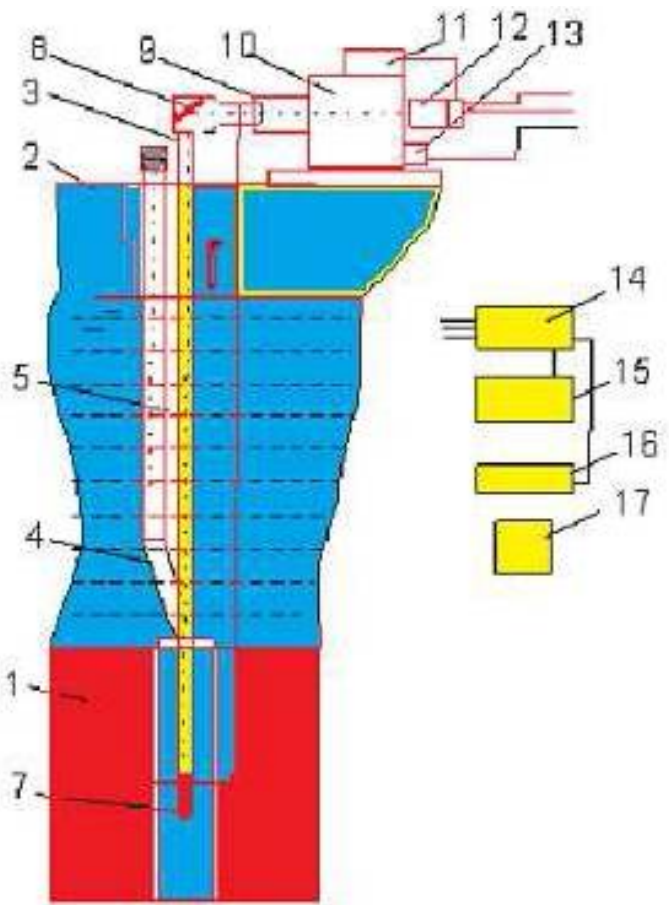

1 - reactor core; 2 - cover of the reactor; 3 - diagnostic channel; 4 - swivel elbow; 5 - transport tube; 6 trumpet-optical fiber; 7 - tested ampulla; 8 - rotary mirror; 9 - lens; 10 - MDR-2; 11 - TES-88; 12 FEU-79; 13 - reversing engine RD-09; 14 - control panel; 15 - VS-22; 16 - analyzer; 17 - recording potentiometer.

Figure 1 - Experimental setup for measuring optical characteristics of the plasma reactor WWR-K.

Experimental study of the optical radiation of the plasma (Figure 1) formed by the products of nuclear reactions has also certain unique features (unlike many other works on the irradiation of materials in the reactor); measurements should be carried out while the reactor is operating at some definite power. The importance of such work (measurements during irradiation) has been repeatedly emphasized [3-6]. Difficulties associated with promotion and performance of such types of a great number of experimental works. The main from these areas follow. The first one is to provide biological protection with minimal loss of light emission and the second is the complexities of registration of the spectrum in terms of the reactor. The first problem is solvable by choosing a specific design of the experimental setup; the second allows us to solve the photoelectric method of recording optical radiation, particularly 
when using a photon counting technique. In the reactor radiation, many optical materials reduce their capacity and reflecting ability and increase their absorbency. It is therefore very important to ensure consistency in the process of the installation of the optical properties, as well as the output window equipment to the cartridge. Biological protection, when the optical output radiation should be held, can be achieved in different ways. Its implementation is based on the difference in interaction with matter reactor radiation "hard" and "soft" $(\mathrm{eV})$ of optical radiation. Property can be used to reflect the optical radiation from specific materials (mirror surfaces), while a "hard" reactor radiation is absorbed in this material, or passes by through them. Usually in spectral diagnostics special ampoules are used made of the cerium glass window [7]. Absolute spectral sensitivity was calibrated using calibrated wolfram lamps SIRSH 8.5-200-1. Measurements of the optical spectra of nuclear excited helium plasmas have shown on the figure 2 .

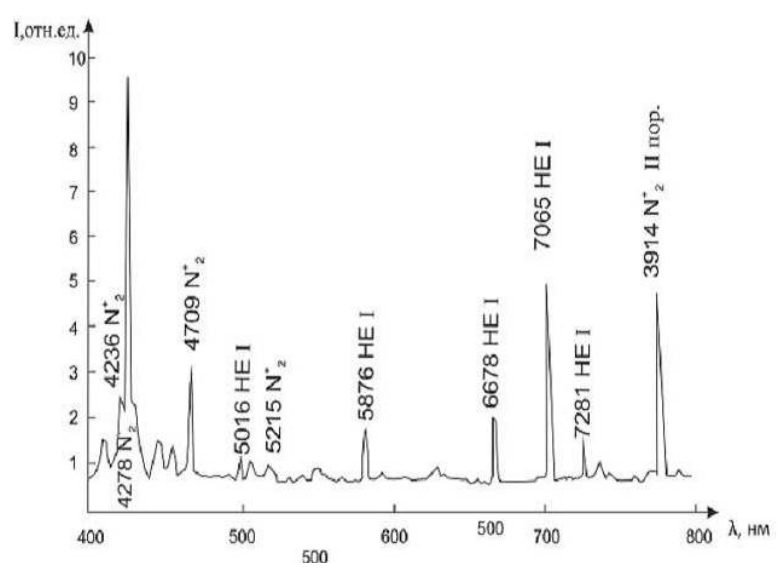

Figure 2 - Spectrogram helium plasma optical radiation ${ }^{3} \mathrm{He}(\mathrm{n}, \mathrm{p}) \mathrm{T}$ at a pressure of $57 \mathrm{kPa}$ containing nitrogen impurity about $2.5^{*} 10^{-4} \%$ under the irradiation of neutron flux $\Phi=10^{14} \mathrm{~cm}^{-2} \mathrm{~s}^{-1}$.

Table 1 shows the values of the measured brightness of the lines in absolute units, as well as the level populations.

Table 1 - Values of measuring the intensity brightness of the optical lines and their levels populations in helium plasma.

\begin{tabular}{|c|c|c|c|c|c|}
\hline Energy levels & $\lambda, \mathrm{nm}$ & $\begin{array}{c}\text { Transition probabili- } \\
\text { ty, } 107 \mathrm{~s}^{-1}\end{array}$ & $I \lambda, 10-7 W_{\mathrm{sm}}-3$ & \multicolumn{2}{|c|}{$\begin{array}{c}\text { Upper and lower population } \\
\text { density }\end{array}$} \\
\hline & & & & $106 \mathrm{~cm}-3$ & $107 \mathrm{~cm}-3$ \\
\hline $21 P-31 S 0$ & 7281 & 1.9 & 37 & 0.71 & 0.88 \\
\hline $33 S 1-23 P$ & 7065 & 2.8 & 206 & 2.63 & 0.53 \\
\hline $31 D 2-21 P 1$ & 6678 & 6.6 & 50.4 & 0.26 & 0.88 \\
\hline $33 D-23 P$ & 5876 & 7.2 & 27.5 & 0.11 & 0.53 \\
\hline $31 P 1-21 S 0$ & 5016 & 1.35 & 18.3 & 0.34 & 500 \\
\hline
\end{tabular}

\section{Helium plasma kinetics. Optical spectra for- mation model}

Characteristic feature of the nuclear excited helium plasma is the lack of optical intensity lines corresponding to transitions from the excited states of atoms with principal quantum number $n \geq 4$. This is due to the fact that the high-pressure collision frequency of the excited atoms with normal becomes comparable to the probability of spontaneous emission. When this type of collisions occur the transfer of excitation between levels with the same principal quantum number and quenching of excited atoms is taking place. It is most likely that the main mechanism of quenching is associative ionization reaction (reaction Hornbeck-Molner). The most at- tention should be paid to the fact that the observed lines corresponding to transitions from the excited states of maximum intensity correspond to transitions from levels $\mathrm{He}(n=3)$ characterized by the smallest cross section for associative ionization. To explain the experimental results, we believe that the most likely following simplified scheme of elementary processes is to be realized. When $3 \mathrm{He}$ ionization by nuclear fragments $(p, T \rightarrow 0.76 \mathrm{Mev})$ is taking place fission fragments produce primary electrons, which also ionize and excite helium atoms (36). Electrons with energies below the excitation threshold relax to low temperatures $\mathrm{Te} \sim 0.1 \mathrm{eV}$, forming the most populated group of electrons plasma electrons [7]. Created in a collision with helium atoms by fast charged particles and primary 
electrons ${ }^{3} \mathrm{He}(n \geq 4)$ atoms during $\sim 1 \mathrm{~nm}$ disappear as a result of associative-ionization of and triple collisions (6-7) . These processes lead to nonradioactive quenching that are not observed in the spectrum line of a helium atom from the states $n \geq 4$. In high-pressure helium plasma conditions are favorable for the formation of molecular ions, such as three-body collisions (9). The resulting molecular ions $\mathrm{He}_{2}^{+}$quickly relax into the ground state $(v=0)$ by collisions with electrons and excited atoms of helium. When $\mathrm{He}_{2}^{+}$recombine with an electron- the value of recombination coefficient may strongly depends on the experimental conditions and may vary tenfold. This is due to the specific nature of the intersection of the terms of the molecular ion and helium auto ionization state. Repulsive branch of the potential interaction curves are not crossing the lower vibration levels of the $\mathrm{He}_{2}^{+}$ ion, which makes it impossible for low-lying $(v \geq 4)$ ions in the process dissotsiative recombination.According to [8] the ground vibration level $\mathrm{He}_{2}^{+}(v=0)$ has the threshold energy $\Delta E \sim 1 \mathrm{eV}$ and obviously with electronic temperature close to $k T_{e} \sim 0.01 \mathrm{eV}, k T_{e} \ll \Delta E$ (where $-\Delta \mathrm{E}-$ the response threshold), the values of lower levels of ions $\mathrm{He}_{2}^{+}(v=0)$ are relatively small $\alpha^{r e c}=3.0 *$ $10^{-11} * T_{e}^{-\frac{3}{2}}[8]$ and significantly more likely it is the transformation them in the three-particle helium ions(10).It should be noted that even at room temperature (after glow discharge) in a weakly ionized helium plasma at sufficiently high pressure there are four varieties of singly charged ions $\mathrm{He}^{+}, \mathrm{He}_{2}^{+}, \mathrm{He}_{3}^{+}, \mathrm{He}_{4}^{+}[7]$. We also state that the fast- est process is the process of dissociative recombination of electrons and complex ions with constant of dissociative recombination coefficient of the order $\sim 10-6 \mathrm{~cm}-3 \mathrm{~s}-1$.

Therefore dissociative recombination is one of the main processes that determine the balance of charged and excited atoms in the low-temperature plasma containing molecular ions .However, because of the thermal instability of the $\mathrm{He}_{3}^{+}$ions $\left(D\left(\mathrm{He}_{2}^{+}-\mathrm{He}\right) \sim 0.17 \mathrm{eV}\right)$ at temperature $\sim 0.01 \mathrm{eV}$ the ionic composition of helium plasma is determined by dimer ions. Because of the small value of the dissociative recombination coefficient the volume neutralization Processes are determined by triple recombination (11-12).According to studies [8-9], about $70 \%$ of the excited states formed in these reactions - are metastable states of helium atoms. The reaction of dissociative recombination of the ion $\mathrm{He}_{3}^{+}$is poorly understood, and for energy conservation reasons, along with the formation of metastable atoms and $\mathrm{He}_{2}^{*}$ molecules in the process of dissociation of $\mathrm{He}_{3}^{+}$complex ion, it is possible its settlements into - singlet and triplet states of helium for the level population with principle quantum number $n=3$. And it is essential that the electron participated in that recombination process should be with an energy of $0.7-1 \mathrm{eV}$. However, due to the large difference in the recombination coefficients $\left(\mathrm{He}_{2}^{+}-\alpha^{r e c}=3.0 * 10^{11} * \mathrm{~T}_{e}^{-3 / 2}, \mathrm{He}_{3}^{+}-\alpha^{r e c}=\right.$ $\left.1.86 * 10^{-6} *\left(0.26 / T_{e}\right)^{1 / 2}\right)$ population of excited states of helium $(n=3)$ will be ruled by the recombination flow of the $\mathrm{He}_{3}^{+}$ions, even if the ratio of $\mathrm{He}_{2}^{+} / \mathrm{He}_{3}^{+}$ions will be two orders of its magnitude.

$$
{ }^{3} \mathrm{He}+(p, T) \rightarrow{ }^{3} \mathrm{He}^{+}+(p, T)_{\varepsilon}+e,
$$

where $\mathrm{T}$ - tritium, $\mathrm{e}$ - electron.

$$
{ }^{3} \mathrm{He}+e_{p} \rightarrow{ }^{3} \mathrm{He}+e_{\varepsilon}+e,
$$

where $\mathrm{e}_{\mathrm{p}}-$ primary electron, $e_{\varepsilon}-$ secondary electron.

$$
\begin{gathered}
{ }^{3} \mathrm{He}+e_{p} \rightarrow{ }^{3} \mathrm{He}^{*}+e_{\varepsilon}+e . \\
{ }^{3} \mathrm{He}^{*}(n \geq 4)+{ }^{3} \mathrm{He} \rightarrow{ }^{3} \mathrm{He}_{2}^{+}+e . \\
{ }^{3} \mathrm{He}(n \geq 4)+{ }^{3} \mathrm{He}+{ }^{3} \mathrm{He} \rightarrow{ }^{3} \mathrm{He}_{2}^{+}+e+{ }^{3} \mathrm{He} . \\
{ }^{3} \mathrm{He}^{+}+2 \mathrm{He} \rightarrow{ }^{3} \mathrm{He}_{2}^{+}+{ }^{3} \mathrm{He}^{*} .
\end{gathered}
$$




$$
\begin{gathered}
{ }^{3} \mathrm{He}_{2}^{+}+\mathrm{He}+\mathrm{He} \rightarrow{ }^{3} \mathrm{He}_{3}^{+}+{ }^{3} \mathrm{He}^{*} . \\
\mathrm{H} e_{2}^{+}+e+e \rightarrow H e^{*}+\mathrm{He}+e . \\
{ }^{3} \mathrm{He}_{2}^{+}+e+\mathrm{He} \rightarrow{ }^{3} \mathrm{He}^{*}+2 \mathrm{He}^{*} . \\
\mathrm{He}_{3}^{+}+e \rightarrow 3 \mathrm{He} e^{*} .
\end{gathered}
$$

The kinetic equations corresponding to (2-11) elementary processes are as follows:

$$
\begin{gathered}
\frac{d\left[\mathrm{He}^{+}\right]}{d t}=S-k 1\left[\mathrm{He}^{+}\right] *[\mathrm{He}]^{2} \\
\frac{d\left[\mathrm{He}_{2}^{+}\right]}{d t}=k 1\left[\mathrm{He} e^{+}\right] *[\mathrm{He}]^{2}-k 2\left[\mathrm{He} e_{2}^{+}\right][\mathrm{He}]^{2}-\alpha_{1}\left[\mathrm{He}_{2}^{+}\right] n_{e} \\
\frac{d\left[\mathrm{He} e_{3}^{+}\right]}{d t}=k 2\left[\mathrm{He}_{2}^{+}\right][\mathrm{He}]^{2}-\alpha_{1}\left[\mathrm{He}_{3}^{+}\right] n_{e} \\
\frac{d n_{e}}{d t}=S-\alpha_{1}\left[\mathrm{He}_{2}^{+}\right] n_{e}-\alpha_{2}\left[\mathrm{He}_{3}^{+}\right] n_{e} \\
\frac{d\left[\mathrm{He}^{*}(n \geq 4)\right]}{d t}=k_{5} n_{e}+\alpha_{2}\left[\mathrm{He}_{3}^{+}\right] n_{e}+\alpha_{1}\left[\mathrm{He}_{2}^{+}\right] n_{e}-k_{3}\left[\mathrm{He}^{*}(n \geq 4)\right]
\end{gathered}
$$

$$
\begin{gathered}
\frac{d\left[H e\left(2^{3} P_{1}\right)\right]}{d t}=k_{13} n_{e}+k_{312}\left[H e^{*}(n \geq 4)+A\left(3^{3} S_{1} \rightarrow 2^{3} P_{1}\right)\left[H e\left(3^{3} S_{1}\right)\right]-\right. \\
-A\left(3^{3} D \rightarrow 2^{3} P\right)\left[H e\left(3^{3} D_{1}\right)\right]-k_{q 13}\left[H e\left(2^{3} P_{1}\right)\right],
\end{gathered}
$$

$\frac{d\left[\mathrm{He}\left(2^{1} S_{0}\right)\right]}{d t}=k_{14} n_{e}+k_{313}\left[\mathrm{He} e^{*}(n \geq 4)+A\left(3^{3} P_{1} \rightarrow 2^{1} S_{0}\right)\left[\mathrm{He}\left(3^{1} P_{1}\right)\right]-k_{q 14}\left[\mathrm{He}\left(2^{1} S_{0}\right)\right]\right.$, $\frac{d\left[\operatorname{He}\left(2^{3} S_{1}\right)\right]}{d t}=k_{15} n_{e}+k_{314}\left[H e^{*}(n \geq 4)+A\left(3^{3} P \rightarrow 2^{3} S_{1}\right)\left[H e\left(3^{3} P_{1}\right)\right]-k_{q 15}\left[H e\left(2^{3} S_{1}\right)\right]\right.$, 


$$
\begin{gathered}
\frac{d I\left(3^{1} S_{0} \rightarrow 2^{1} P_{1}\right)}{d t}=h v\left(3^{1} S_{0} \rightarrow 2^{1} P_{1}\right) * A\left(3^{1} S_{0} \rightarrow 2^{1} P_{1}\right) * \frac{d\left[\mathrm{He}\left(3^{0} S_{1}\right)\right]}{d t}, \\
\frac{d I\left(3^{1} P_{1} \rightarrow 2^{1} S_{0}\right)}{d t}=h v\left(3^{1} P_{1} \rightarrow 2^{1} S_{0}\right) * A\left(3^{1} P_{1} \rightarrow 2^{1} S_{0}\right) * \frac{d\left[\mathrm{He}\left(3^{1} P_{1}\right)\right]}{d t} \\
\frac{d I\left(3^{1} D_{2} \rightarrow 2^{1} P_{1}\right)}{d t}=h v\left(3^{1} D_{2} \rightarrow 2^{1} P_{1}\right) * A\left(3^{1} D_{2} \rightarrow 2^{1} P_{1}\right) * \frac{d\left[H e\left(3^{1} D_{2}\right)\right]}{d t}, \\
\frac{d I\left(3^{1} S_{1} \rightarrow 2^{3} P_{1}\right)}{d t}=h v\left(3^{3} S_{1} \rightarrow 2^{3} P_{1}\right) * A\left(3^{3} S_{1} \rightarrow 2^{3} P_{1}\right) * \frac{d\left[H e\left(3^{3} S_{1}\right)\right]}{d t}, \\
\frac{d I\left(3^{3} P \rightarrow 2^{3} S_{1}\right)}{d t}=h v\left(3^{3} P \rightarrow 2^{3} S_{1}\right) * A\left(3^{3} P \rightarrow 2^{3} S_{1}\right) * \frac{d\left[H e\left(3^{3} P_{1}\right)\right]}{d t} \\
\frac{d I\left(3^{3} D \rightarrow 2^{3} P\right)}{d t}=h v\left(3^{3} D \rightarrow 2^{3} P\right) * A\left(3^{3} D \rightarrow 2^{3} P\right) * \frac{d\left[H e\left(3^{3} D\right)\right]}{d t},
\end{gathered}
$$

energy from $i$ to $j$ state.

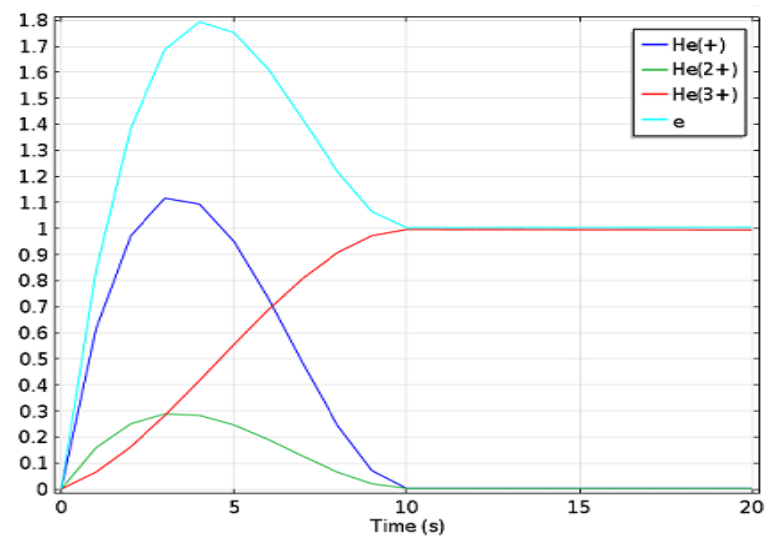

Figure 3 - Time dependence of helium and electron concentrations in plasma.

In the experiments [10] done by one of the authors of the paper (Kunakov S.K.) all lines were observed, except (38) which was out the region of experimental optics registration tools and all optical transitions intensities in equation (12-32) are presented as follows:

$$
\begin{aligned}
& I\left(3^{1} S_{0} \rightarrow 2^{1} P_{1}\right), h v=7281 \mathrm{~nm}, \\
& I\left(3^{3} S_{1} \rightarrow 2^{3} P_{1}\right), h v=7065 \mathrm{~nm}, \\
& I\left(3^{1} D_{2} \rightarrow 2^{1} P_{1}\right), h v=6678 \mathrm{~nm}
\end{aligned}
$$

$$
\begin{gathered}
I\left(3 D \rightarrow 2^{3} P\right), h v=5876 \mathrm{~nm}, \\
I\left(3^{1} P_{1} \rightarrow 2^{1} S_{0}\right), h v=5016 \mathrm{~nm}, \\
I\left(3^{3} P \rightarrow 2^{3} S_{1}\right), h v=3888 \mathrm{~nm} .
\end{gathered}
$$

On the Figure 3 the time dependence of helium ions and electrons density is calculated based on kinetic model described by equations (12-32).

Good experimental (Table 1) and theoretical calculations agreement of optical intensities in helium plasma is demonstrated on Figure 4.

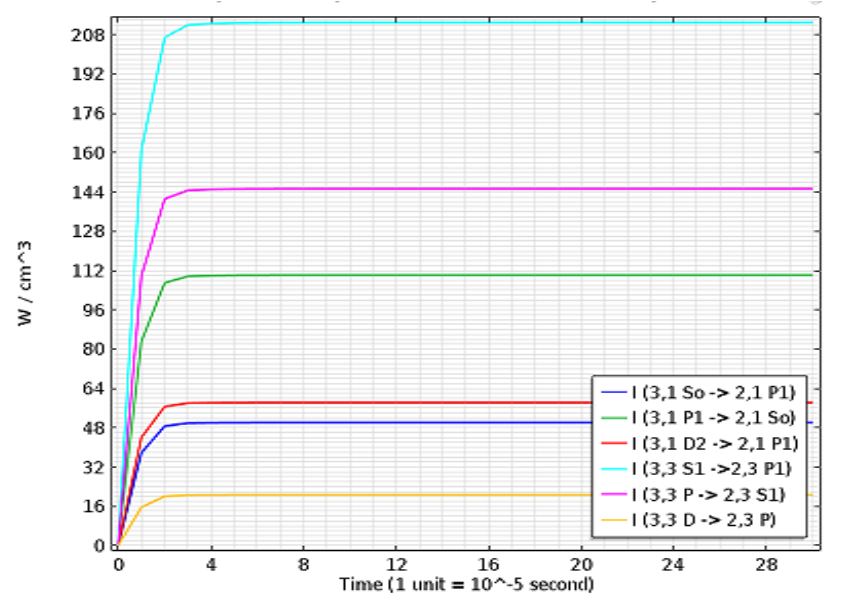

Figure 4 - Time dependence of optical intensities in helium plasma.

\section{Conclusion}

For the quantitative determination of the proportion of the recombination flux populating levels $(\mathrm{n}=$ 3 ) is necessary to know the distribution function of 
the electron energy. Despite of the large number of studies on various processes in nuclear - induced plasma $3 \mathrm{He}(\mathrm{n}, \mathrm{p})$ [3-6] in the literature, there are no data on the spectral energy distribution of its optical radiation. Authors are usually limited to the individual measurements of the radiation intensity nuclear-induced plasma in order to search for new laser media. However, a detailed study of the elementary processes taking place in the nuclear - induced plasma, application of heterochromic photometry(absolute spectra),to our opinion, is necessary because only the measurement of the absolute brightness of the line performance and absorption can give accurate information about the distribution of the population of the various states. In addition, these measurements allow a quantitative analysis of the efficiency of conversion of nuclear energy into light gas mixtures and might be used as active media sources of optical radiation.

\section{References}

1. Kunakov S.K. Izmerenie absolyutnyh intensivnostei spectralnyh linii opticheskogo diapazona izlucheniya plazmy $3 \mathrm{He}(\mathrm{n}, \mathrm{p}) \mathrm{T}$. Al-Farabi Kazakh National university, vestnik, seriya fizicheskaya. 2010. - Vol.33. - N. 2.

2. Kunakov S.K., Redkin A.V. Izmerenie absolyutnyx intensivnostei spektralnyx lini opticheskogo izlucheniya plazmy $3 \mathrm{He}(\mathrm{n}, \mathrm{p}) \mathrm{T}$, obrazuyushhei syapri obluchenii $3 \mathrm{He}$ nejtronnym potokom stacionarnogo yadernogo reaktora VVR-K.// TVT. - 1986. Vol.26. - N. 4. - P.633-638.
3. Tomizo I., Toshimitsu M.Monter Carlo Calculations of motion of electrons in Helium // Journal of the Physical Society of Japan. - 1960. - Vol.15. N. 9. - P.1675-1680.

4. Benjamin S.W.,Geoge H.M. Monte Carlo simulations of Radiation-Induced Plasmas // Nuclear Science and Engineering. - 1973. - Vol.52. P.130-141.

5. LeffertC.B., Reese D.B., Jamerson F.E. Noble Gas Plasmas Produced by Fission Fragments// J.Appl.Phys. - 1966. - Vol.37., - P.133-142.

6. Nguyen D.H.,Grossmsan L.M. Ionization by Fission Fragments Escaping from Source Medium// Nucl.Sci. andEngin. - 1967. - Vol.30. - P.233-241.

7. Guyot J.C.,Miley G.H.,Verdeyen J.T. Application of a Two Region Heavy Charged Particle Model to Noble Gas Plasmas Induced by Nuclear Radiation //Nucl.Sci. andEngin. - 1972.-Vol.48. P.372-386

8. Kunakov S.K., Son E.E. Probe Diagnostics of Nuclear Excited Plasma of Uranium Hexafluoride // High Temperature. - 2010. - Vol.48. -N. 6. P.789-805.

9. Kunakov S.K.,Redkin A.V. Studies of the luminescent characteristics of helium mercury mixture excited by a nuclear reactor // Journal of Applied Spectroscopy. - 1990.- Vol.52.-N. 2. P.221-225.

10. Lakoba I.S., Yakovlenko S.I. Aktivnye sredy kompleksnyx lazerov. Obzor // Kvantovaya elektronika. -1980. -Vol.7. - N. 4. - P.1140-1176.

11. Michal G. Classical Theory of Atomic Collissions. I. Theory of Inelastic Collisions // Physical Review. - 1965. - Vol.138. - N. 2A. - P. 336-358. 